\title{
Oleanolic acid suppresses vascular smooth muscle cell proliferation by increasing lincRNA-p21 expression
}

\author{
DI HAN ${ }^{1}$, XUEJUAN ZHANG ${ }^{1}$, JIETAO ZHANG ${ }^{1}$, XIAOZI GUO ${ }^{1}$, \\ YU ZHENG $^{1}$, SHIHUA SUI ${ }^{2}$ and JIAPING ZHENG $^{2}$ \\ ${ }^{1}$ Department of General Medicine, The Affiliated Hospital of Qingdao University, Qingdao, Shandong 266031; \\ ${ }^{2}$ Department of Neurology, People's Hospital of Rizhao, Rizhao, Shandong 276800, P.R. China
}

Received June 25, 2015; Accepted July 7, 2016

DOI: $10.3892 / 01.2016 .5096$

\begin{abstract}
Arteriosclerosis poses a significant risk to human health and involves the thickening and hardening of the walls of arteries. Accumulated evidence demonstrates that aberrant proliferation of vascular smooth muscle cells (VSMCs) accounts for the onset and progression of arteriosclerosis. Suppression of their proliferation has been demonstrated to be an effective anti-arteriosclerosis strategy. Long non-coding RNAs (lncRNAs) have recently been observed to be implicated in the proliferation of VSMCs and arteriosclerosis. In this study, we observed that oleanolic acid (OA), a natural compound from plants, inhibited the proliferation of VSMCs. The expression of lincRNA-p21, an arteriosclerosis-associated IncRNA, was demonstrated to be elevated by OA treatment. Suppression of lincRNA-p21 rescued the effect of OA on the proliferation of VSMCs. Collectively, targeting lncRNA is a promising strategy for arteriosclerosis prevention and treatment, and OA ameliorates arteriosclerosis by increasing lncRNA levels.
\end{abstract}

\section{Introduction}

As a leading cause of mortality, arteriosclerosis presents a significant risk to human health. It has been responsible for 16,000,000 heart attacks and 5,800,000 strokes in 2008 in the USA. Although advances have been achieved in medicine, the prevalence of arteriosclerosis remains (1). Studies have revealed that arteriosclerosis is characterized by narrowed lumen filled with thickened smooth muscle cells, which hinders the circulation of blood flow (1). These vascular smooth muscle cells (VSMCs) are believed to translocate from the tunica media to the tunica intima, following rapid and

Correspondence to: Professor Jiaping Zheng, Department of Neurology, People's Hospital of Rizhao, 126 Tai'an Road, Rizhao, Shandong 276800, P.R. China

E-mail: jiapingzhengrz@126.com

Key words: oleanolic acid, long non-coding RNA, vascular smooth muscle cell uncontrolled proliferation (2). Based on the above knowledge, the suppression of VSMCs is considered to be a feasible therapeutic strategy against arteriosclerosis.

Further studies have revealed that the activation of specific molecular signaling pathways, including ERK, Notch, Wnt and NF- $\mathrm{NB}$, plays an key role in the aberrant proliferation of VSMCs (3). Natural compounds that are capable of interrupting the activation of these signaling pathways have been demonstrated to exert an inhibitory effect on VSMC proliferation. For instance, ligustrazine was demonstrated to attenuate VSMC proliferation by suppressing ERK and p38 MAPK signaling (4). Similarly, the natural compound reinioside C suppresses the proliferation of smooth muscle cells by inhibiting the NADPH oxidase-ROS-ERK1/2-NF- $\mathrm{B}-\mathrm{AP}-1$ pathway (5). Long non-coding RNA (lncRNA), a type of nuclear RNA molecule that cannot be translated into a protein product, has previously been confirmed to inhibit the proliferation of arteriosclerosis-associated VSMCs (6). However, it is unclear whether lncRNA is an effective therapeutic target for VSMC-directed anti-arteriosclerosis treatment.

In this study, we observed that oleanolic acid (OA), a natural compound widely distributed in a wide range of plants with various verified bioactivities, exerts an inhibitory effect on the proliferation of VSMCs in rats by increasing lincRNA-p21 expression. Our findings provide evidence that lncRNA mediates the effect of the natural compound on VSMC biology.

\section{Materials and methods}

Primary rat smooth muscle cell culture. Rat smooth muscle cells were used in the present study, which was approved by the ethics committee of Qingdao University (Qingdao, China). The isolation and culturing procedure has been described previously (7). PDGF-bb (30 ng/ml, PHG0041, Thermo Fisher Scientific, Inc., Waltham, MA, USA) was added to the culture to stimulate the proliferation of VSMCs.

Chemicals. OA was obtained from Sigma-Aldrich (St. Louis, MO, USA) and preserved in dimethyl sulfoxide (DMSO). In the study, OA $(20,40$ or $80 \mu \mathrm{M})$ was added to the culture of VSMCs, and then the proliferation rates of VSMCs were determined at the indicated time points. In the control groups, DMSO was added to the culture. 
Cell counting. The number of cells was determined with a Millipore Scepter ${ }^{\mathrm{TM}}$ handheld automated cell counter (Merck Millipore, Darmstadt, Germany) according to the instructions provided at the indicated time points. The counting was performed at least three times. The bars represent the means \pm standard deviation.

Bromodeoxyuridine (BrdU) assay. BrdU assay was conducted to detect the proliferation rates of VSMCs. The protocols are briefly described as follows. Culture medium was removed and replaced with $\mathrm{BrdU}$ labeling solution at $37^{\circ} \mathrm{C}$ for $2 \mathrm{~h}$. Then the labeling solution was removed and cells were washed twice with phosphate-buffered saline (PBS). One milliliter of $3.7 \%$ formaldehyde was used to fix the cells for $15 \mathrm{~min}$ at room temperature, then cells were washed twice with PBS. Next, $1 \mathrm{ml}$ Triton ${ }^{\circledR}$ X-100 permeabilization buffer was added to each well and the cells were incubated for $20 \mathrm{~min}$ at room temperature. One milliliter of antibody staining buffer was subsequently added to each well, and anti-BrdU primary antibody was used to treat cells overnight at room temperature. Fluorescently labeled secondary antibody was then added and the cells were incubated for $1 \mathrm{~h}$. Finally, the cells were observed under an IX71-A12FL/PH microscope (Olympus Corporation, Tokyo, Japan). BrdU ${ }^{+}$cells were counted and recorded for the calculation of percentages.

lncRNA detection. Quantitative polymerase chain reaction (qPCR) was performed to detect the lincRNA-p21 expression level. Total RNA was extracted from the cells with TRIzol solution (Sigma-Aldrich). Following reverse transcription reaction, qPCR was performed using TaqMan ${ }^{\circledR} 2 \mathrm{X}$ Universal PCR master mix (Applied Biosystems; Thermo Fisher Scientific, Inc.) on a CFX96 ${ }^{\mathrm{TM}}$ Real-Time PCR detection system (Bio-Rad Laboratories, Inc., Hercules, CA, USA) supplied with analytical software. The primers used are as follows: forward: TCCTTCTTGTGGTTGTGACT; reverse: GGGCTC AAGGGATCGGCCTG.

Immunoblot assays. Total protein was extracted from cells, followed by $1 \%$ polyacrylamide gel electrophoresis-based separation. After $0.45 \mu \mathrm{m}$ membrane transferring and $5 \%$ milk blocking, samples were incubated with the following primary antibodies: anti-p-IKK $\beta, 1: 500$ (BD Biosciences, San Jose, CA, USA); anti-total IKK $\beta$ antibody, 1:1,000 (BD Biosciences); anti-IкB $\alpha$ antibody, 1:1,000 (Cell Signaling Technology, Inc., Danvers, MA, USA; anti-IкB $\beta$ antibody, 1:1,000 (Cell Signaling Technology); anti-p65 antibody, 1:1,000 (Cell Signaling Technology), and subsequently appropriate secondary antibodies. Following the treatment of substrates, the bands were visualized using a Tanon-4200 imaging system (Tiangen, China).

Statistical analysis. Statistical analysis in our study was performed using a two-tailed Student's t-test. $\mathrm{P}<0.05$ and $\mathrm{P}<0.01$ were considered to indicate a significant and a very significant statistical difference between two groups, respectively.

\section{Results}

Oleanolic acid suppresses proliferation of VSMCs in a doseand time-dependent manner. First, we employed cell counting

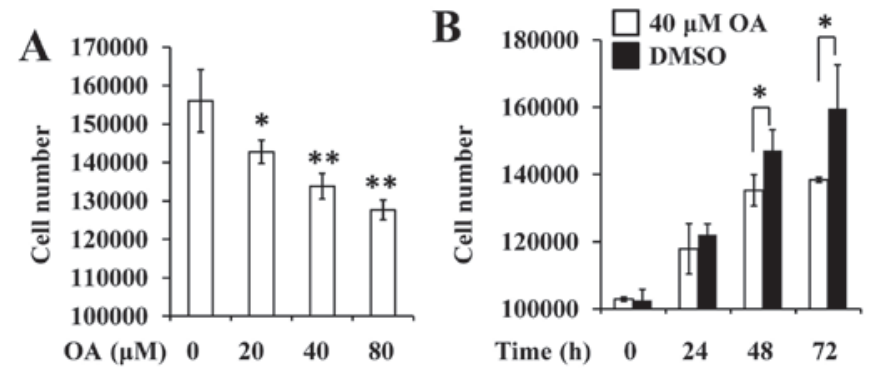

Figure 1. Oleanolic acid (OA) reduced the number of vascular smooth muscle cells (VSMCs) in a dose- and time-dependent manner. (A) Rat VSMCs were treated with OA $(0,20,40$ or $80 \mu \mathrm{M})$ for $48 \mathrm{~h}$. Cells were directly counted with a Millipore Scepter ${ }^{\mathrm{TM}}$ handheld automated cell counter. (B) VSMCs were treated with OA $(40 \mu \mathrm{M})$. Cells were directly counted with the Millipore Scepter cell counter at the indicated timepoints $(0,24,48$ and $72 \mathrm{~h}) .{ }^{*} \mathrm{P}<0.05$, ${ }^{* * *} \mathrm{P}<0.01$ compared with control.
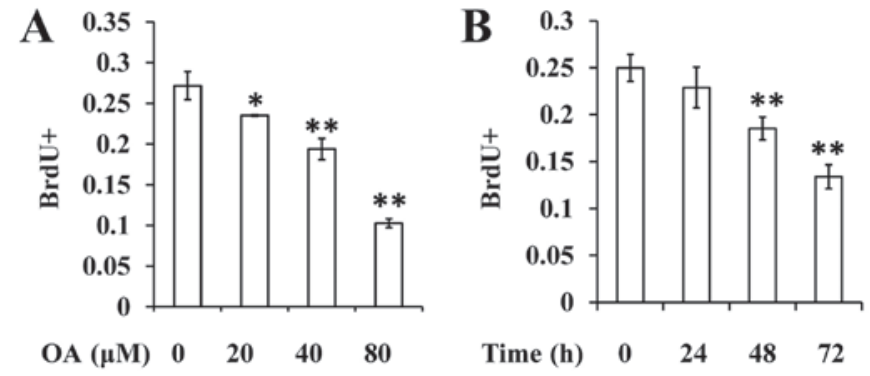

Figure 2. Oleanolic acid (OA) suppresses the proliferation of vascular smooth muscle cells (VSMCs) in a dose- and time-dependent manner. (A) Rat VSMCs were treated with OA $(0,20,40$ or $80 \mu \mathrm{M})$ for $48 \mathrm{~h}$. BrdU assay was performed to determine the proliferation rates. (B) VSMCs were treated with OA $(40 \mu \mathrm{M})$. BrdU assay was performed to determine the proliferation rates at the indicated timepoints $(0,24,48$ and $72 \mathrm{~h}) .{ }^{*} \mathrm{P}<0.05,{ }^{* *} \mathrm{P}<0.01$ compared with control.

and BrdU assay to detect the effect of OA on rat VSMCs. The results revealed that the number of VSMCs was significantly reduced $48 \mathrm{~h}$ after treatment with the various concentrations of OA (Fig. 1A). Similarly, $40 \mu \mathrm{M}$ OA was observed to decrease the number of cells at the indicated time points $(24,48$ and 72 h, Fig. 1B).

BrdU assay was conducted to determine the effect of OA on the proliferation of VSMCs. The results revealed that OA is able to decrease the percentage of $\mathrm{BrdU}^{+}$cells in a dose- and time-dependent manner (Fig. 2A and B).

LincRNA-p21 expression is restored in VSMCs by OA. Given the fact that lincRNA-p21 downregulation contributes to the uncontrolled proliferation of VSMCs, we aimed to elucidate whether OA affected the expression of lincRNA-p21. The data revealed that OA significantly increases the expression of lincRNA-p21 in VSMCs in a dose- and time-dependent manner (Fig. 3A and B).

$N F-\kappa B$ signaling is suppressed in VSMCs by OA. A previous study reported that the lincRNA-p21 level is inversely correlated with the activation of NF- $\mathrm{\kappa B}$ in rheumatoid arthritis, implying a regulatory effect of lincRNA-p21 on NF- $\kappa \mathrm{B}$ signaling (8). Thus, we employed immunoblot analysis to determine the activation of the NF- $\kappa \mathrm{B}$ pathway in OA-treated 


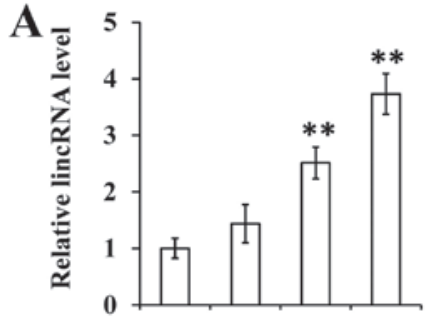

OA $(\mu \mathrm{M}) \quad 0 \quad 20 \quad 40 \quad 80$
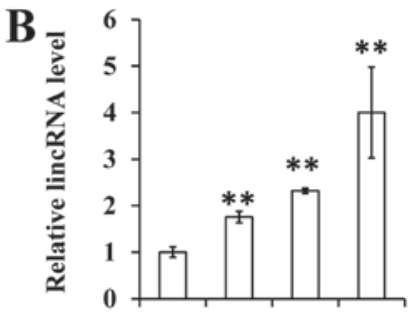

$\begin{array}{lllll}\text { Time (h) } & 0 & 24 & 48 & 72\end{array}$

Figure 3. lincRNA-p21 expression was restored in vascular smooth muscle cells (VSMCs) by oleanolic acid (OA). (A) Rat VSMCs were treated with OA $(0,20,40$ or $80 \mu \mathrm{M})$ for $48 \mathrm{~h}$. lincRNA-p21 expression was determined by quantitative polymerase chain reaction (qPCR) assay. U6 was used as an endogenous reference. (B) VSMCs were treated with OA $(40 \mu \mathrm{M})$. lincRNA-p21 expression was determined by qPCR assay at the indicated timepoints $(0,24,48$ and $72 \mathrm{~h})$. U6 was used as an endogenous reference. ${ }^{* *} \mathrm{P}<0.01$ compared with control.

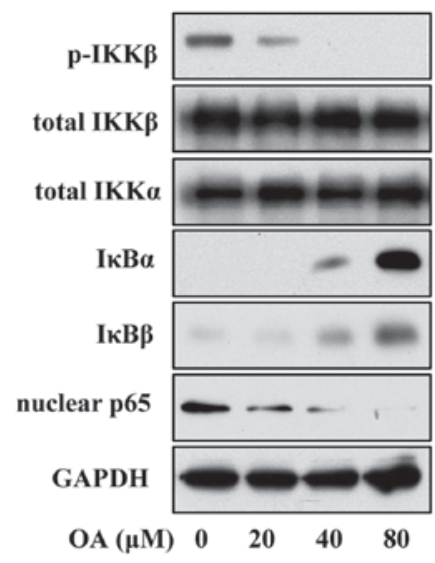

Figure 4. NF- $\mathrm{KB}$ signaling was suppressed in vascular smooth muscle cells (VSMCs) by oleanolic acid (OA). Rat VSMCs were treated with OA $(0,20$ 40 or $80 \mu \mathrm{M}$ ) for $48 \mathrm{~h}$. The expression of key regulators of NF- $\kappa \mathrm{B}$ signaling was detected by immunoblot analysis. GAPDH was used as a loading control.

VSMCs. The data revealed that OA treatment induced lower expression of phosphorylated IKK, increased the level of IкB $\beta$ and reduced the abundance of nuclear p65, suggesting that OA suppressed the activation of the NF- $\kappa \mathrm{B}$ pathway (Fig. 4).

Suppression of lincRNA-p21 rescues the effect of OA on the proliferation of VSMCs. Given the finding that OA treatment led to increased expression of lincRNA-p21, we subsequently aimed to investigate the significance of this event for the inhibitory effect of OA on VSMC proliferation. We infected cells with a lentiviral vector expressing shRNA targeting lincRNA-p21 (sh-lincRNA-p21; Fig. 5A). Following this treatment, NF- $\kappa \mathrm{B}$ signaling in VSMCs was observed to resist the effect of OA (Fig. 5B). The suppression of lincRNA-p21 appeared to eliminate the inhibitory effect of OA on the proliferation of VSMCs (Fig. 6A and B).

\section{Discussion}

OA has been well documented to exert various bioactivities. Its effect on VSMCs was studied by Feng et al (9). The ERK/Nrf2/HO-1 signaling pathway was confirmed to mediate

A

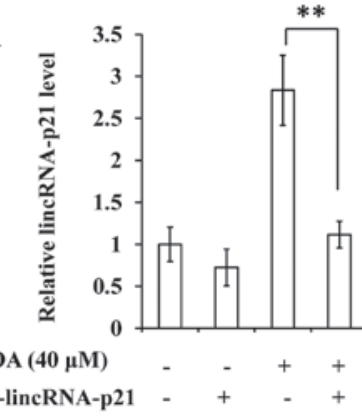

B

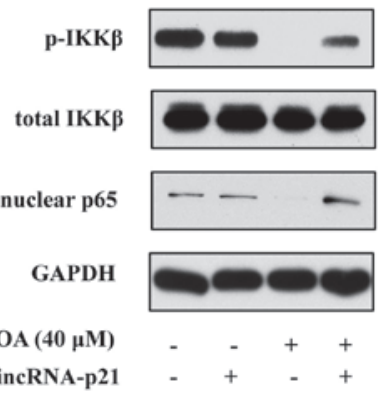

Figure 5. Suppression of lincRNA-p21 leads to reactivation of NF- $\kappa$ B signaling. (A) Rat vascular smooth muscle cells (VSMCs) were treated with oleanolic acid (OA; $40 \mu \mathrm{M})$ and/or sh-lincRNA-p21 for $48 \mathrm{~h}$. lincRNA-p21 expression was determined by quantitative polymerase chain reaction assay. U6 was used as an endogenous reference. (B) Rat VSMCs were treated with OA $(40 \mu \mathrm{M})$ and/or sh-lincRNA-p21 for $48 \mathrm{~h}$. The expression of key regulators of NF- $\kappa \mathrm{B}$ signaling was detected by immunoblot analysis. GAPDH was used as a loading control. ${ }^{* *} \mathrm{P}<0.01$ compared with control.
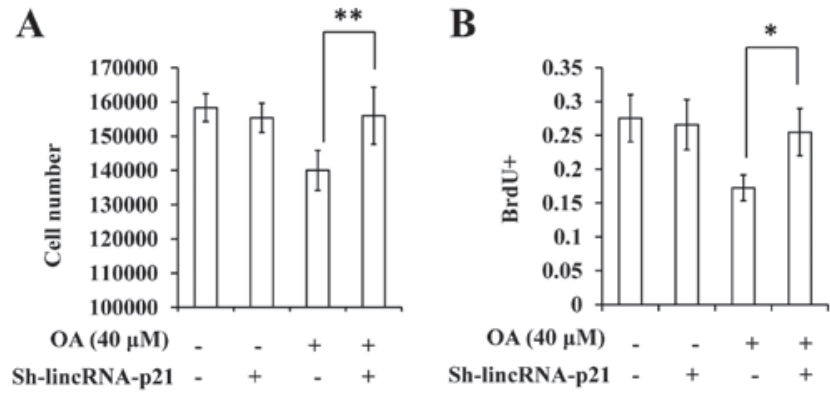

Figure 6. Suppression of lincRNA-p21 rescues the effect of oleanolic acid (OA) on the proliferation of vascular smooth muscle cells (VSMCs). (A) Rat VSMCs were treated with OA $(40 \mu \mathrm{M})$ and/or sh-lincRNA-p21 for $48 \mathrm{~h}$. Cells were directly counted with a Millipore Scepter ${ }^{\mathrm{TM}}$ handheld automated cell counter. (B) Rat VSMCs were treated with OA $(40 \mu \mathrm{M})$ and/or sh-lincRNA-p21 for $48 \mathrm{~h}$. BrdU assay was performed to determine the proliferation rates. ${ }^{*} \mathrm{P}<0.05,{ }^{* *} \mathrm{P}<0.01$ compared with control.

the protective effect of $\mathrm{OA}$ on $\mathrm{H}_{2} \mathrm{O}_{2}$-induced oxidative damage in VSMCs. However, the effect of OA on the proliferation of VSMCs had not been studied previously. In this study, we provided evidence that OA reduces the proliferation rates of rat VSMCs, which play a significant role in the progression of arteriosclerosis.

Although the aberrant activation of certain molecular signaling pathways has been established to be crucial for arteriosclerosis, a previous study added lncRNA to the major regulators for the onset and progression of this vascular disease (6). lincRNA-p21, which is underexpressed in arteriosclerosis patients, suppresses the proliferation of VSMCs by promoting the transcription of numerous p53 targets. Restoring p53 expression has been demonstrated to ameliorate the severity of arteriosclerosis. However, it is unclear whether lincRNA-p21 is an effective therapeutic target for arteriosclerosis. Our study provided evidence that lincRNA-p21 mediates the effect of OA on the proliferation of VSMCs. VSMC proliferation is assumed to contribute to the progression of arteriosclerosis, implying that lincRNA-p21 may be a promising target for anti-arteriosclerosis therapy.

The significance of the restoration of lincRNA-p21 by $\mathrm{OA}$ is not limited to the treatment of cardiovascular diseases. 
Cancer, which is the second highest cause of human mortality, is resistant to chemotherapy due to the low specificity and numerous side effects of current antitumor drugs. A number of natural compounds, including $\mathrm{OA}$, have been demonstrated to suppress tumor growth without significant side effects (10-14). $\mathrm{OA}$ is therefore considered to be a promising compound in the clinical application of cancer treatment. The association between lincRNA-p21 and cancer has also been studied previously (15). LincRNA-p21 suppresses the malignant phenotypes of cancer through multiple pathways (16-18). More notably, lincRNA-p21 may be used to determine the clinical prognosis of colon cancers (19). However, it has not been determined whether lincRNA-p21 is associated with the antitumor activity of OA. Further studies should be conducted in order to address this issue.

Taken together, we observed that OA suppresses the proliferation of VSMCs by increasing the expression of lincRNA-p21. Our findings indicated that targeting lincRNA-p21 may be an effective strategy for anti-arteriosclerosis treatment, and that OA is a candidate compound for arteriosclerosis therapy.

\section{References}

1. Sun AS and Renaud M: Enhancement of 5'-nucleotidase activity of human leukemic cells after fractionation: implications for cancer and aging. Mutat Res 219: 295-302, 1989.

2. Chaabane C, Coen M and Bochaton-Piallat ML: Smooth muscle cell phenotypic switch: implications for foam cell formation. Curr Opin Lipidol 25: 374-379, 2014.

3. Rudijanto A: The role of vascular smooth muscle cells on the pathogenesis of atherosclerosis. Acta Med Indones 39: 86-93, 2007.

4. Yu L, Huang X, Huang K, Gui C, Huang Q and Wei B: Ligustrazine attenuates the platelet-derived growth factor-BB-induced proliferation and migration of vascular smooth muscle cells by interrupting extracellular signal-regulated kinase and P38 mitogen-activated protein kinase pathways. Mol Med Rep 12: 705-711, 2015

5. Hong D, Bai YP, Shi RZ, Tan GS, Hu CP and Zhang GG: Inhibitory effect of reinioside $\mathrm{C}$ on vascular smooth muscle cells proliferation induced by angiotensin II via inhibiting NADPH oxidase-ROS-ENK1/2-NF-kappaB-AP-1 pathway. Pharmazie 69: 698-703, 2014.
6. Wu G, Cai J, Han Y, Chen J, Huang ZP, Chen C, Cai Y, Huang H, Yang Y, Liu Y, et al: LincRNA-p21 regulates neointima formation, vascular smooth muscle cell proliferation, apoptosis, and atherosclerosis by enhancing p53 activity. Circulation 130: 1452-1465, 2014

7. Qin X, Qiu C and Zhao L: Maslinic acid protects vascular smooth muscle cells from oxidative stress through Akt/Nrf2/HO-1 pathway. Mol Cell Biochem 390: 61-67, 2014.

8. Spurlock CF III, Tossberg JT, Matlock BK, Olsen NJ and Aune TM: Methotrexate inhibits NF- $\mathrm{KB}$ activity via long intergenic (noncoding) RNA-p21 induction. Arthritis Rheumatol 66: 2947-2957, 2014.

9. Feng J, Zhang P, Chen X and He G: PI3K and ERK/Nrf2 pathways are involved in oleanolic acid-induced heme oxygenase-1 expression in rat vascular smooth muscle cells. J Cell Biochem 112: 1524-1531, 2011.

10. Liu J, Zheng L, Ma L, Wang B, Zhao Y, Wu N, Liu G and Lin X: Oleanolic acid inhibits proliferation and invasiveness of Kras-transformed cells via autophagy. J Nutr Biochem 25: 1154-1160, 2014

11. Liu J, Wu N, Ma LN, Zhong JT, Liu G, Zheng LH and Lin XK: p38 MAPK signaling mediates mitochondrial apoptosis in cancer cells induced by oleanolic acid. Asian Pac J Cancer Prev 15: 4519-4525, 2014.

12. Liu J, Zheng L, Zhong J, Wu N, Liu G and Lin X: Oleanolic acid induces protective autophagy in cancer cells through the JNK and mTOR pathways. Oncol Rep 32: 567-572, 2014.

13. Liu J, Zheng L, Wu N, Ma L, Zhong J, Liu G and Lin X: Oleanolic acid induces metabolic adaptation in cancer cells by activating the AMP-activated protein kinase pathway. J Agric Food Chem 62: 5528-5537, 2014.

14. Liu J, Wu N, Ma L, Liu M, Liu G, Zhang Y and Lin X: Oleanolic acid suppresses aerobic glycolysis in cancer cells by switching pyruvate kinase type M isoforms. PLoS One 9: e91606, 2014.

15. Gutschner T and Diederichs S: The hallmarks of cancer: A long non-coding RNA point of view. RNA Biol 9: 703-719, 2012.

16. Yang F, Zhang H, Mei Y and Wu M: Reciprocal regulation of HIF-1 $\alpha$ and lincRNA-p21 modulates the Warburg effect. Mol Cell 53: 88-100, 2014.

17. Wang G, Li Z, Zhao Q, Zhu Y, Zhao C, Li X, Ma Z, Li X and Zhang Y: LincRNA-p21 enhances the sensitivity of radiotherapy for human colorectal cancer by targeting the Wnt/ $\beta$-catenin signaling pathway. Oncol Rep 31: 1839-1845, 2014.

18. Dimitrova N, Zamudio JR, Jong RM, Soukup D, Resnick R, Sarma K, Ward AJ, Raj A, Lee JT, Sharp PA and Jacks T: LincRNA-p21 activates p21 in cis to promote Polycomb target gene expression and to enforce the G1/S checkpoint. Mol Cell 54: 777-790, 2014

19. Zhai H, Fesler A, Schee K, Fodstad O, Flatmark K and Ju J: Clinical significance of long intergenic noncoding RNA-p21 in colorectal cancer. Clin Colorectal Cancer 12: 261-266, 2013. 